ANNALES

POLONICI MATHEMATICI

$\mathrm{XI}$ (1962)

\title{
Évaluations des solutions de l'équation linéaire du' type parabolique à coefficients non bornés
}

\author{
par M. KRzYżá́skI (Kraków)
}

1. Dans un travail récent, publié en collaboration avec A. Szybiak [4] nous avons déterminé la solution fondamentale de l'équation linéaire parabolique

(1) $\mathscr{F}[u] \equiv \sum_{i, j=1}^{m} a_{i j}(t, X) u_{x_{i x}}^{\prime \prime}+\sum_{k=1}^{m} b_{k}(t, X) u_{x_{k}}^{\prime}+c(t, X) u-u_{t}^{\prime}=0$,

$X$ étant un point variable de coordonnées $x_{1}, x_{2}, \ldots, x_{m}$ de l'espace euclidien $\mathcal{E}^{m}$ à $m$ dimensions. Les coefficients $a_{i j}(t, X)$ et $b_{k}(t, X)$ étaient supposés bornés et assez réguliers dans une couche $e: X \in \mathcal{E}^{m}, S<t<T$ (dans le présent travail nous supposons que $S=0$ ) et, par hypothèse, il existait un nombre $A_{0}>0$ tel que

$$
q(A)=\sum_{i, j=1}^{m} a_{i j}(t, X) \lambda_{i} \lambda_{j} \geqslant A_{0} \mid \Lambda_{i}^{2}
$$

pour tout point $(t, X) \in \Theta$ et pour tout vecteur $\Lambda\left(\lambda_{1}, \lambda_{2}, \ldots, \lambda_{m}\right)$.

Le coefficient $\boldsymbol{c}(t, X)$ était supposé continu dans $e$, lipschitzien par rapport à $X$ et on supposait l'existence des nombres constants $A>0$ et $B>0$ tels qu'on en̂t dans $e$

$$
|c(t, X)| \leqslant A^{2}|X|^{2}+B\left({ }^{1}\right), \quad \text { où } \quad|X|=\left(\sum_{i=1}^{m} x_{i}^{2}\right)^{1 / 2} .
$$

Ces hypothèses concernant le coefficient $c(t, X)$ étaient appelées brièvement hypothèses $(\mathrm{H})$.

La solution fondamentale $U(t, X ; s, Y)$ de l'équation (1), qui a été déterminée dans [4], est continue dans un ensemble $\delta: X \in \mathcal{E}^{m}, \boldsymbol{Y} \in \mathcal{E}^{m}, 0 \leqslant s$ $<t<T, 0<t-s<T(A)$, le nombre $T(A) \leqslant T$ dépendant en général de $A$; elle admet des dérivées des deux premiers ordres par rapport aux variables $x_{i}$ et $y_{i}$ et des dérivées du premier ordre par rapport aux variables $t$ et $s$ continues à l'intérieur de cet ensemble. La fonction $U(t, X ; s, \bar{Y})$ satisfait à l'équation adjointe à (1) en tant que fonction de $s$ et $\boldsymbol{Y}$.

(1) J'ai démontré qu'on peut déterminer la solution fondamentale jouissant des mêmes propriétés si l'on a $c(t, X) \leqslant A^{2}|X|^{2}+B$. Ce résultat n'est pas encore publié. 
Soit $\varphi(X)$ une fonction continue et de classe $\mathbb{E}_{2}\left({ }^{2}\right)$ dans $\mathcal{E}^{m}$. L'intégrale

$$
u(t, X)=\int_{\varepsilon^{m}} U(t, X ; 0, Y) \varphi(Y) d Y
$$

constitue la solution unique (voir [3]) de (1) régulière $\left(^{3}\right)$ dans une couche $C$ : $0 \leqslant t<T^{\prime}, X \in \mathcal{E}^{m}\left(T^{\prime} \leqslant T\right)$, de classe $E_{2}$ et satisfaisant à la condition initiale

$$
u(0, X)=\varphi(X) \quad \text { pour } \quad X \in \varepsilon^{m} \text {. }
$$

Soient $c_{1}(t, X)$ et $c_{2}(t, X)$ deux fonctions satisfaisant aux hypothèses (H). Posons

$$
\mathscr{F}_{0}[u]=\sum_{i, j=1}^{m} a_{i j}(t, X) u_{x_{i} x_{j}}^{\prime \prime}+\sum_{k=1}^{m} b_{k}(t, X) u_{x_{k}}^{\prime}-u_{t}^{\prime}
$$

et soient $U_{\boldsymbol{r}}(t, X ; s, Y)(r=1,2)$ les solutions fondamentales des équations

$$
\mathscr{F}_{0}[u]+c_{r}(t, X) u=0 .
$$

D'après le théorème 7 de $[4]$, si $c_{1}(t, X) \leqslant c_{2}(t, X)$ dans $e$, on a aussi $U_{1}(t, X ; s, Y) \leqslant U_{2}(t, X ; s, \bar{Y})$ dans $\delta$. Le théorème reste valable lorsque $T=\infty$ et les fonctions $U_{r}(t, X ; s, Y)$ existent pour $X \in \mathcal{C}^{m}, Y \in \mathcal{C}^{m}$ et $0<t-s<\infty$.

Dans le présent travail nous allons établir certaines évaluations des solutions de l'équation (1) suivant l'allure du coefficient $\left.c(t, X){ }^{4}\right)$.

2. Dans le cas particulier de l'équation

$$
\Delta w-w_{t}^{\prime}+\left(\alpha^{2}|X|^{2}+\beta\right) w=0
$$

$\left(\Delta w=\sum_{i=1}^{m} w_{x_{i} x_{i}}^{\prime \prime}\right), \alpha>0$ et $\beta$ étant deux nombres constants, la solution fondamentale a été déterminée effectivement par A. Szybiak (voir [4]), à savoir

$$
\begin{aligned}
& W(t, X ; s, Y)=\left[\frac{2 \pi}{\alpha} \sin 2 \alpha(t-s)\right]^{-m / 2} \exp \left[-\frac{\alpha}{2}\left(|X|^{2}+|Y|^{2}\right) \operatorname{ctg} 2 \alpha(t-s)+\right. \\
& \left.+\alpha X \cdot Y \sin ^{-1} 2 \alpha(t-s)+\beta(t-s)\right] \quad\left(X \in \mathcal{E}^{m}, Y \in \mathcal{E}^{m}, 0<t-s<\pi / 2 \alpha\right)
\end{aligned}
$$

(a) Nous appelons $E_{\alpha}$ une classe de fonctions $f(X)$ qui sont définies dans $\mathcal{E}^{m}$ (ou dans un ensemble non borné de $\left.\mathcal{E}^{m}\right)$ et $\mathbf{y}$ satisfont à l'inégalité

$$
|f(X)| \leqslant M \exp \left(K|X|^{a}\right) \text {, }
$$

où $M$ et $K$ sont deux nombres constants positifs qui dépendent de la fonotion $f(X)$. Dans la suite nous appelerons aussi fonction de classe $E_{\alpha}$ une fonction de $X$ et de qui est de classe $E_{a}$ en tant que fonction de $X$, les nombres $M$ et $K$ ne dépendant pas de $t$.

(a) C'est-à-dire continue dans $O$ et admettant des dérivées du second ordre par rapport aux variables $x_{i}$ et une dérivée du premier ordre par rapport à la variable $t$ continues à l'intériear de $C$.

(4) Ces évaluations ont été prévues par A. Szybiak, mais il n’a pas précisé les hypothèses permettant de les établir. (où $X \cdot Y=\sum_{k=1}^{m} x_{k} y_{k}$ ). Soit $\mu$ un nombre positif. La fonction

$$
w_{\mu}(t, X)=\mu \int_{\varepsilon^{m}} W(t, X ; 0, Y) d Y
$$

constitue, d'après la formule (2), la solution (unique) de (3) régulière et de classe $E_{2}$ dans la couche $0 \leqslant t<\pi / 4 a, X \in \mathcal{E}^{m}$ et satisfaisant à la condition initiale $w_{\mu}(0, X)=\mu$. En effectuant des calculs convenables, on obtient pour $w_{\mu}(t, X)$ l'expression suivante

$$
w_{\mu}(t, X)=\mu(\cos 2 \alpha t)^{-m / 2} \exp \left[\frac{\alpha}{2}|X|^{2} \operatorname{tg} 2 \alpha t+\beta t\right] .
$$

Considérons l'équation

$$
\Delta u-u_{t}^{\prime}+c(t, X) u=0,
$$

où la fonction $c(t, X)$ est définie dans une couche $0 \leqslant t<t_{0}, X \in \mathcal{E}^{m}$ (il peut arriver que $t_{0}=\infty$ ) et y satisfait aux hypothèses (H). Supposons que l'on ait

$$
c(t, X) \geqslant \alpha^{2}|X|^{2}+\beta
$$

$\alpha>0$ et $\beta$ étant des nombres constants. Soit $u(t, X)$ une solution de l'équation (5) régulière et de classe $E_{2}$ dans une couche $0 \leqslant t<T^{\prime \prime}, X \in \mathcal{C}^{m}$ $\left(T^{\prime \prime} \leqslant \min \left(t_{0}, \pi / 4 \alpha\right)\right.$ ) et telle que $u(0, X) \geqslant \mu$. Il résulte du théorème 7 de [4] (que nous venons de citer) et de la formule (2) qu'on a $u(t, X) \geqslant$ $\geqslant w_{\mu}(t, X)$; par conséquent, il existe deux nombres positifs $\bar{M}$ et $\bar{K}$ tels que l'on a

$$
u(t, X) \geqslant \bar{M} \exp \left(\bar{K}|X|^{2} \operatorname{tg} 2 a t\right) \quad\left(t \in\left(0, T^{\prime \prime}\right), X \in \mathcal{E}^{m}\right) .
$$

3. Il en est tout autrement, si le coefficient $c(t, X)$ de l'équation (5) vérifie (outre les hypothèses (H)) l'inégalité

$$
c(t, X) \leqslant-\alpha^{2}|X|^{2}+\beta,
$$

$\alpha>0$ et $\beta$ étant des nombres constants. Dans le cas particulier $c(t, X)$ $=-\alpha^{2}|X|^{2}+\beta$ la solution fondamentale de (5) a été déterminée effectivement par A. Szybiak dans la note [5], à savoir

$$
\begin{aligned}
& \text { (7) } \quad V(t, X ; s, Y)=\left[\frac{2 \pi}{\alpha} \sinh 2 \alpha(t-s)\right]^{-m / 2} \times \\
& \times \exp \left[-\frac{\alpha}{2}\left(|X|^{2}+|Y|^{2}\right) \operatorname{ctgh} 2 \alpha(t-s)+\alpha X \cdot Y \sinh ^{-1} 2 \alpha(t-s)+\beta(t-s)\right]
\end{aligned}
$$

et cette solution fondamentale est définie dans l'ensemble $0<t-s<\infty$, $X \in \mathcal{E}^{m}, Y \in \mathcal{E}^{m}$. La solution $v_{\mu}(t, X)$ de $(5)$, régulière et de classe $E_{\mathbf{2}}$ pour 
$t \geqslant 0$, satisfaisant à la condition initiale $v_{\mu}(0, X)=\mu, \mu$ étant un nombre constant positif, calculée d'après la formule (2), s'exprime alors par la formule

$$
v_{\mu}(t, X)=\mu(\cosh 2 \alpha t)^{-m / 2} \exp \left[-\frac{\alpha}{2}|X|^{2} \operatorname{tgh} 2 \alpha t+\beta t\right]
$$

et on a $\lim _{|X| \rightarrow \infty} v_{\mu}(t, X)=0$ (exponentiellement) pour $t>0$ fixé.

Observons que, la fonction tgh $2 a t$ étant bornée, l'allure de $v_{\mu}(t, X)$ pour $t \rightarrow \infty$, lorsque $X$ est fixé, est la même que celle de l'expression

$$
v^{(0)}(t, X)=(\cosh 2 \alpha t)^{-m / 2} e^{\beta t}=e^{(\beta-m a) t}\left[\frac{1+e^{-4 a t}}{2}\right]^{-m / 2} ;
$$

or l'allure de $v^{(0)}(t, X)$ pour $t \rightarrow \infty$ dépend du signe de la différence $\beta-m \alpha\left(^{(5)}\right.$.

Supposons maintenant que le coefficient $c(t, X)$ de l'équation (5) soit une fonction satisfaisant aux hypothèses $(\mathrm{H})$ et à l'inégalité (6). I résulte du théorème $7 \mathrm{de}[4]$ et des formules (2) et (8) que, $u(t, X)$ étant une solution de l'équation (5) régulière et de classe $E_{2}$ pour $t \geqslant 0, X \in \varepsilon^{m}$ et telle que la fonction $\varphi(X)=u(0, X)$ soit bornée dans $\mathcal{E}^{m}$, on a l'inégalité

$$
|u(t, X)| \leqslant M(\cosh 2 \alpha t)^{-m / 2} \exp \left[-\frac{\alpha}{2}|X|^{2} \operatorname{tgh} 2 \alpha t+\beta t\right]
$$

où $M$ est la borne supérieure de $|\varphi(X)|$ dans $\mathcal{E}^{m}$.

4. Nous allons voir qu'on peut démontrer un théorème beaucoup plus général. Considérons une équation parabolique de la forme (1) et supposons que ses coefficients soient définis pour $t>0, X \in \mathcal{E}^{m}$, les coefficients $a_{t y}(t, X)(i, j=1, \ldots, m)$ y étant bornés et la forme

$$
\mathcal{A}[A]=\sum_{i, j=1}^{m} a_{i j}(t, X) \lambda_{i} \lambda_{i j}
$$

y étant définie positive: Quant aux coefficients $b_{k}(t, X)$, nous supposons l'existence des nombres $A_{1} \geqslant 0$ et $B_{1} \geqslant 0$ tels que l'on ait

$$
\left|b_{k}(t, X)\right| \leqslant A_{1}|X|+B_{1} \quad \text { pour } \quad t>0, \quad X \in \varepsilon^{m} .
$$

Enfin, nous supposons que le coefficient $c(t, X)$ satisfait à l'inégalité

$$
c(t, X) \leqslant-a^{2}|X|^{2}+\beta \quad \text { pour } \quad t>0, \quad X \in \mathcal{C}^{m} .
$$

(5) L'influence de l'expression $\beta-m a$ sur l'allure de la solution fondamentale de l'équation de la forme (5), aveo $o(t, X)=-\alpha^{2}|X|^{2}+\beta$, a été disoutée dans le travail [5] de A. Szybiak.
Ceci posé, nous allons démontrer le théorème suivant

THÉoRł̀me. Soit $u(t, X)$ une solution de l'équation (1) régulière et de classe $E_{2}$ pour $t \geqslant 0, X \in \mathcal{E}^{m}$, telle que la fonction $\varphi(X)=u(0, X)$ soit bornée dans $\mathcal{E}^{m}$. On a l'inégalité

$$
|u(t, X)| \leqslant M \exp \left[-\lambda|X|^{2} \operatorname{tgh} \gamma t+\nu t\right],
$$

$M$ étant la borne supérieure de $|\varphi(X)|$ dans $\mathcal{E}^{m}, \gamma>0, \lambda>0$ et $\nu$ étant des nombres dépendant des coefficients $a_{i j}(t, X)$, des nombres $A_{1}$ et $B_{1}$ qui interviennent dans l'inégatité (10) et des nombres $\alpha$ et $\beta$.

Démonstration. Soit $\mathfrak{A}$ le plus petit nombre positif tel que l'on ait

$$
\sum_{i, j=1}^{m} a_{i j}(t, X) \lambda_{i} \lambda_{j} \leqslant \mathfrak{U} \sum_{k=1}^{m} \lambda_{k}^{2}
$$

pour $t>0, X \in \mathcal{E}^{m}$ et pour tout vecteur $\Lambda\left(\lambda_{1}, \ldots, \lambda_{m}\right)$. Il résulte de (10) que l'on peut déterminer un nombre non négatif $\mathfrak{B}$ de sorte que l'on ait

$$
\left|\sum_{k=1}^{m} b_{k}(t, X) x_{k}\right| \leqslant \mathfrak{B}\left(m+|X|^{2}\right) \quad \text { pour } \quad t>0, \quad X \in \mathcal{E}^{m}
$$

supposons que $\mathfrak{B}$ soit le plus petit de tels nombres.

Nous introduisons une nouvelle fonction inconnue $v(t, X)$ en posant

$$
u(t, X)=v(t, X) \exp \left[-\vartheta(t)|X|^{2}+\omega(t)\right],
$$

$\vartheta(t)$ et $\omega(t)$ étant deux fonctions non négatives et de classe $C^{1}$ pour $t \geqslant 0$, s'annulant pour $t=0$, la fonction $\vartheta(t)$ y étant en outre bornée. Ces fonctions seront choisies convenablement dans la suite. La fonction $v(t, X)$ constitue pour $t>0, X \in \mathcal{E}^{m}$ une solution de l'équation de la forme

$$
\sum_{i, j=1}^{m} a_{i j}(t, X) v_{x_{i x j}}^{\prime \prime}+\sum_{k=1}^{m} \bar{b}_{k}(t, X) v_{x_{k}}^{\prime}+\bar{c}(t, X) v-v_{t}^{\prime}=0,
$$

où l'on a

$$
\begin{aligned}
\bar{b}_{k}(t, X)= & b_{k}(t, X)-4 \vartheta(t) \sum_{i=1}^{m} a_{i k}(t, X) x_{i}, \\
\bar{c}(t, X)= & \vartheta^{\prime}(t)|X|^{2}+4(\vartheta(t))^{2} \sum_{i, j=1}^{m} a_{i j}(t, X) x_{i} x_{j}- \\
& -2 \vartheta(t) \sum_{j=1}^{m} a_{j j}(t, X)-2 \vartheta(t) \sum_{k=1}^{m} b_{k}(t, X) x_{k}-\omega^{\prime}(t)+c(t, X) .
\end{aligned}
$$

Il en résulte qu'il existe deux nombres $\bar{A}_{1}$ et $\bar{B}_{1}$ tels que

(16) $\left|\bar{b}_{k}(t, X)\right| \leqslant \bar{A}_{1}|X|+\bar{B}_{1} \quad$ pour $\quad t>0, \quad X \in \mathcal{E}^{m} \quad(k=1, \ldots, m)$. 
D'autre part, en posant

$$
a_{0}=\min _{j \leqslant m}\left[\inf _{t>0, X \in \mathcal{E}^{m}} a_{j j}(t, X)\right],
$$

on a, en vertu de (13) et (14)

(17) $\quad \bar{c}(t, X) \leqslant\left[\vartheta^{\prime}(t)+4 \mathfrak{A}(\vartheta(t))^{2}+2 \mathfrak{B} \vartheta(t)-\alpha^{2}\right]|X|^{2}+$

$$
+2 m\left(\mathfrak{B}-a_{0}\right) \vartheta(t)+\beta-\omega^{\prime}(t) \text {. }
$$

Nous allons choisir les fonctions $\vartheta(t)$ et $\omega(t)$ de façon à obtenir l'inégalité $\bar{c}(t, X) \leqslant 0$, ce qui permettra d'appliquer le théorème 2 de [3] à la fonction $v(t, X)$. Nous posons

$$
\vartheta(t)=\lambda \operatorname{tgh} 4 \mathfrak{A} \lambda t \quad \text { pour } \quad t \geqslant 0,
$$

$\lambda$ étant la racine positive de l'équation

on a alors

$$
4 \mathfrak{U} \lambda^{2}+2 \mathfrak{B} \lambda=\alpha^{2}
$$

(19) $\vartheta^{\prime}(t)+4 \mathfrak{A}(\vartheta(t))^{2}+2 \mathfrak{B} \vartheta(t)=4 \mathfrak{A} \lambda^{2}+2 \mathfrak{B} \lambda \operatorname{tgh} 4 \mathfrak{A} \lambda \leqslant \alpha^{2}$ pour $t \geqslant 0$.

Nous posons ensuite

$$
(20) \quad \omega(t)=\beta t+2 m\left(\mathfrak{B}-a_{0}\right) \int_{0}^{t} \vartheta(\tau) d \tau
$$

$$
=\beta t+\frac{m}{2 \mathfrak{U}}\left(\mathfrak{B}-a_{0}\right) \log \cosh 4 \mathfrak{U} \lambda t ;
$$

on a alors, d'après (17) et (19), l'inégalité $\bar{c}(t, X) \leqslant 0$ pour $t>0, X \in \mathcal{E}^{m}$.

Comme $v(0, X)=u(0, X)$ et par suite $|v(0, X)| \leqslant M$ pour $X \in \mathcal{E}^{m}$, il résulte $d u$ théorème 2 de [3] que l'on a

$$
|v(t, X)| \leqslant M . \quad \text { pour } \quad t \geqslant 0, \quad X \in \mathcal{E}^{m} .
$$

On en déduit, en tenant compte de la définition de la fonction $v(t, X)$, l'inégalité

(21) $\quad|u(t, X)| \leqslant M(\cosh 4 \mathfrak{U} \lambda t)^{\varrho} \exp \left[-\lambda|X|^{2} \operatorname{tgh} 4 \mathfrak{U} \lambda t+\beta t\right]$,

avec

$$
\varrho=\frac{m}{2 \mathfrak{A}}\left(\mathfrak{B}-a_{0}\right) .
$$

Oomme $\cosh y \leqslant e^{y}$ pour $y \geqslant 0$, on en tire l'évaluation (12) avec

$$
\nu=\beta+2 m \lambda\left(\mathfrak{B}-a_{0}\right), \quad \gamma=4 \mathfrak{A} \lambda .
$$

De l'inégalité (21) on déduit certaines conséquences concernant l'allure de la fonction $u(t, X)$ pour $t \rightarrow \infty$, notamment on a $\lim _{t \rightarrow \infty} u(t, X)=0$, si $\nu=\beta+2 m \lambda\left(\mathfrak{B}-a_{0}\right)<0$.
Lorsque $b_{k}(t, X) \equiv 0 \quad(k=1, \ldots, m)$ et par suite $\mathfrak{B}=0$, il vient

$$
\lambda=\frac{\alpha}{2 \sqrt{\mathfrak{A}}}
$$

alors l'inégalité (21) prend la forme

(22) $|u(t, X)| \leqslant M(\cosh 2 \sqrt{\mathfrak{A}} \alpha t)^{-m a_{0} / 2 \mathfrak{2}} \exp \left[-\frac{a}{2 \sqrt{\mathfrak{A}}}|X|^{2} \operatorname{tgh} 2 \sqrt{\mathfrak{A}} \alpha t+\beta t\right]$.

En particulier si $a_{i j}(t, X)=0$ pour $i \neq j$ et $a_{i i}(t, X)=1(i, j=1, \ldots, m)$, on a $\mathfrak{A}=1$ et l'inégalité (22) se réduit à l'inégalité (9) du $\mathrm{n}^{0} 3$.

5. Nous avons établi certaines évaluations des solutions de l'équation parabolique en admettant leur existence.

Si les coefficients de l'équation (1) satisfont aux hypothèses faites au $\mathrm{n}^{0} 4$ et si $c(t, X) \leqslant \alpha^{2}|X|^{2}+\beta$ dans $C$, où $\alpha$ et $\beta$ sont deux nombres constants, on peut démontrer l'existence d'une solution de l'équation (1) régulière et de classe $E_{2}$ dans une couche $0 \leqslant t \leqslant \widetilde{T}, X \in \mathcal{E}^{m}$ et satisfaisant à la condition initiale $u(0, X)=\varphi(X)$, où $\varphi(X)$ est une fonction continue, de classe $E_{2}$ dans $\mathcal{E}^{m}$, en appliquant le procédé exposé dans les travaux [1] et [2]. Cependant, il est alors nécessaire de faire l'hypothèse suivante: $D$ étant un domaine cylindrique, séparé de l'intérieur de $C$ par une surface $|X|=R$ ( $R$ étant un nombre positif), il existe une solution de l'équation (1) régulière dans la fermeture $\bar{D}$ de $D$ et prenant des valeur données sur la surface latérale de $D$ et pour $t=0$.

Si les coefficients $a_{i j}(t, X)$ et $b_{k}(t, X)(i, j, k=1, \ldots, m)$ sont bornés pour $t>0, X \in \mathcal{E}^{m}$, la forme $\mathscr{A}[\Lambda]$ étant supposée définie positive, et si le coefficient $c(t, X)$ est borné supérieurement, on démontre de même l'existence d'une solution de l'équation (1) régulière et de classe $E_{1}$ dans ce demi-espace et satisfaisant à la condition initiale $u(0, X)=\varphi(X)$, où $\varphi(X)$ est une fonction de classe $E_{1}$, à condition de modifier l'hypothèse précédente en admettant pour $D$ un domaine cylindrique séparé du demiespace $t>0, X \in \mathcal{E}^{m}$ par une surface $X=R$. $\AA$ cet effet, on reprend le même procédé en appliquant la fonction introduite au $n^{\circ} 9$ de [1] au lieu du diviseur amortissant $H$ (voir [1] et [2]).

\section{Travaux cités}

[1] M. Krzyżański, Sur les solutions de l'équation linéaire du type parabotique [1] [2] - Sur les solutionss de l'équation linéaire du type parabolique déterminées par les conditions initiales (note complémentaire), Ann. Soc. Polon. Math. 20 (1947), p. 7-9.

[3] - Certaines inégalités relatives aux solutions de l'équation parabolique linéaire normale, Bull. Acad. Polon. Sc., Sér. des so. math., astr. et phys. 7 (1959), p. 131-135. 
[4] M. Krzyżański et A. Szybiak, Construction et étude de la solution fondamentale de l'équation linéaire du type parabolique dont le dernier coefficient est non borné, Note I e II, Atti Accad. Naz. Lincei, el. Sc. fis., mat. e natur., ser. VIII, 27 (1959), p. 1-10.

[5] A. Szybiak, On the Asymptotic Behaviour of the Solutions of the Equation Ond 7 (1959) p. 183-186.

\section{Sur l'allure asymptotique des solutions de l'équation différentielle ordinaire du second ordre}

par T. D£omko (Katowice)

Il existe une classe d'équations différentielles ordinaires du second ordre de la forme

$$
u^{\prime \prime}(t)+g(t, u(t))=0,
$$

possédant de nombreuses propriétés de l'équation différentielle linéaire $u^{\prime \prime}(t)+a(t) u(t)=0$.

Je vais démontrer dans cette note les propriétés que voici: les solutions de l'équation (1) existent dans tout l'intervalle $\left\langle t_{0}, \infty\right.$ ), elles sont bornées, oscillantes ou non oscillantes, elles possèdent des asymptotes, les suites de leurs extrêmes sont monotones, etc.

En particulier, dans le cas où $g(t, u)=a(t) f(u)$, je généralise le résultats obtenus à l'équation différentielle $\left(k(t) u^{\prime}\right)^{\prime}+l(t) / k(t) f(u)=0$.

En outre, les théorèmes demontrés entraînent, comme propositions, certains théorèmes établis par les auteurs [1], [2], [7], [8], [9], [12], [13], [15], [16].

Le trait caractéristique de cette note sera l'emploi de simples et brèves méthodes de démonstration.

J'ai l'honneur de remercier M. A. Bielecki pour ses précieuses remaiques et suggestions, et M. Z. Opial pour son aide dans la rédaction de cette note.

I. Dans cette partie de la note nous allons démontrer un théorème qui, entre autres, assure l'existence des solutions de l'équation (1) dans l'intervalle $t \geqslant t_{0}$.

THस́ов亡̀ME 1. Considérons le système d'équations différentielles

$$
x_{i}^{\prime}(t)=f_{i}\left(t, x_{1}(t), \ldots, x_{n}(t)\right), \quad i=1, \ldots, n,
$$

dans lequel les fonctions $f_{i}$ sont définies et continues dans le domaine $D\left\{t \geqslant t_{0},-\infty<x_{i}<+\infty, i=1, \ldots, n\right\}$. Nous supposons qu'il existe une fonction $w(t, u)$ définie, continue et non négative dans le domaine $D_{1}\left\{t \geqslant t_{0}, u \geqslant 0\right\}$ et telle que l'équation différentielle

$$
u^{\prime}(t)=w(t, u(t)),
$$

ait toutes ses solutions $u(t)$ définies dans tout l'intervalle $t \geqslant t_{0}$. 